\title{
Contribution à l'étude de la stabilité du front de taille d'un tunnel en milieu cohérent
}

\author{
Contribution to the analysis of the face stability \\ of a tunnel excavated in cohesive ground
}

\author{
Eric LECA \\ Laboratoire Central des Ponts et Chaussées* \\ Luc DORMIEUX \\ Laboratoire de Mécanique des Solides, Ecole Polytechnique
}

Rev. Franç. Géotech. n 61, pp. 5-16 (décembre 1992)

\section{Résumé}

Le problème de la stabilité du front de taille d'un tunnel a fait l'objet de nombreuses études au cours des vingt dernières années en raison de la construction d'ouvrages dans des conditions de plus en plus difficiles. En particulier la théorie du calcul à la rupture a permis d'aboutir à un encadrement de la pression de soutènement à appliquer au niveau du front de taille pendant les travaux pour assurer la stabilité du tunnel.

L'étude décrite dans cet article est destinée à améliorer les estimations théoriques du risque d'instabilité au front de taille dans le cas d'un tunnel circulaire creusé à faible profondeur dans un terrain purement cohérent. L'accent est mis sur l'approche par l'extérieur. Les mécanismes examinés permettent de tenir compte du caractère tridimensionnel du problème abordé ainsi que des risques d'instabilités globales et locales. Les résultats conduisent à penser que les différences constatées entre bornes supérieures et inférieures des chargements potentiellement supportables pourraient provenir de la surestimation des capacités de résistance du terrain, qui résultent de leur caractérisation par un critère de TRESCA (autorisant la mise en traction du terrain).

\section{Abstract}

Tunnel face stability has received special consideration over the past twenty years, due to the construction of tunnels in increasingly difficult conditions. In particular, yield calculation principles have been used to find a bracketted estimate of the tunnel pressure that should be used to stabilize the face during construction.

The purpose of the present study is to improve existing methods used to estimate the face stability of shallow circular tunnels driven in purely cohesive soils. New upper bound estimates are considered. The failure mechanisms considered allow to account for the three-dimensional nature of the problem and include local as well as global failure conditions. The results support the idea that the differences between estimated upper and lower bound solutions could result from overestimated ground resistance properties, consecutive to the use of the TRESCA criterion (which allows for tensile stresses in the ground). 


\section{INTRODUCTION}

Le problème de la stabilité du front de taille d'un tunnel en milieu cohérent a été abordé pour la première fois par BROMS et BENNEMARK (1967). Plusieurs auteurs se sont intéressés à ce problème par la suite. Les travaux les plus récents portent sur l'utilisation de modèles réduits en centrifugeuse et l'application de la théorie du calcul à la rupture (SALENÇON, 1990).

Le problème posé étant caractérisé par un nombre fini de paramètres de chargements, Panalyse de la stabilité consiste à déterminer le domaine des sollicitations supportables dans l'espace de ces paramètres de chargements. La théorie du calcul à la rupture propose une méthode pour encadrer ce domaine en approchant sa frontière respectivement par l'intérieur et par l'extérieur.

Dans cet esprit, les travaux de DAVIS et al. (1980) ont permis de préciser les conditions d'utilisation du critère de stabilité établi par BROMS et BENNERMARK (1967), en mettant notamment en évidence l'effet de la profondeur relative du tunnel, c'est-à-dire du rapport entre la couverture du tunnel et son diamètre. Néanmoins leurs résultats ne permettent pas d'aboutir à une quantification précise du risque d'instabilité du fait de l'écart important existant entre les approches par l'intérieur et l'extérieur. Cet écart constitue évidemment un obstacle à l'élaboration de méthodes de dimensionnement basées sur une analyse théorique de la stabilité.

Selon ces auteurs, l'analyse d'essais d'effondrement en centrifugeuse semblait par ailleurs indiquer que les conditions de rupture observées expérimentalement (SCHOFIELD, 1980) étaient proches de celles déduites de l'approche par l'intérieur. Il était donc naturel de tenter d'améliorer les approches par l'extérieur disponibles. C'est le propos de la présente étude qui s'inscrit dans le cadre de la théorie du calcul à la rupture et examine un ensemble de nouveaux mécanismes de rupture virtuels en géométrie tridimensionnelle.

\section{CADRE DE L'ÉTUDE}

La modélisation de la géométrie adoptée pour l'analyse de la stabilité du front de taille d'un tunnel à faible profondeur est représentée sur la figure 1. On considère un tunnel circulaire, de diamètre $\mathrm{D}$ construit sous une couverture $C$, c'est-à-dire que son axe est situé à une profondeur $\mathrm{H}=\mathrm{C}+\frac{\mathrm{D}}{2}$.

Une pression uniforme $\sigma_{\mathrm{T}}$ est maintenue au niveau du front de taille pour assurer sa stabilité. Jusqu'à une distance au front de taille égale à $\mathrm{P}$, la paroi du tunnel est soumise à la même pression $\sigma_{T}$. Au-delà, elle est tenue par un soutènement parfaitement rigide. La surface du massif dans lequel le tunnel est excavé est soumise à une pression de surface $\sigma_{5}$, qui peut représenter la charge transmise par une fondation souple ou encore le poids d'une couche de sol située au dessus de la cote $\mathrm{H}$, mesurée à partir de l'axe du tunnel. La donnée du poids volumique $\gamma$ du sol, supposé homogène dans tout le massif achève de définir la sollicitation considérée. Les capacités de résistance du terrain sont supposées homogènes et isotropes. Elles sont définies par un critère de Tresca, de cohésion $\mathrm{C}_{\mathrm{u}}$.

Le problème peut être caractérisé par cinq paramè. tres adimensionnels : $\frac{\mathrm{C}}{\mathrm{D}}, \frac{\mathrm{P}}{\mathrm{D}}, \sigma_{\mathrm{s}} / \mathrm{C}_{\mathrm{u}}, \sigma_{\mathrm{T}} / \mathrm{C}_{\mathrm{u}}$ et

$\gamma \mathrm{D} / \mathrm{C}_{\mathrm{u}}$. Pour la suite, on se placera dans le cas particulier $P / D=0$, ce qui constitue une hypothèse raisonnable pour l'étude d'un tunnel creusé au bouclier. On aura recours à la notion classique de facteur de charge :

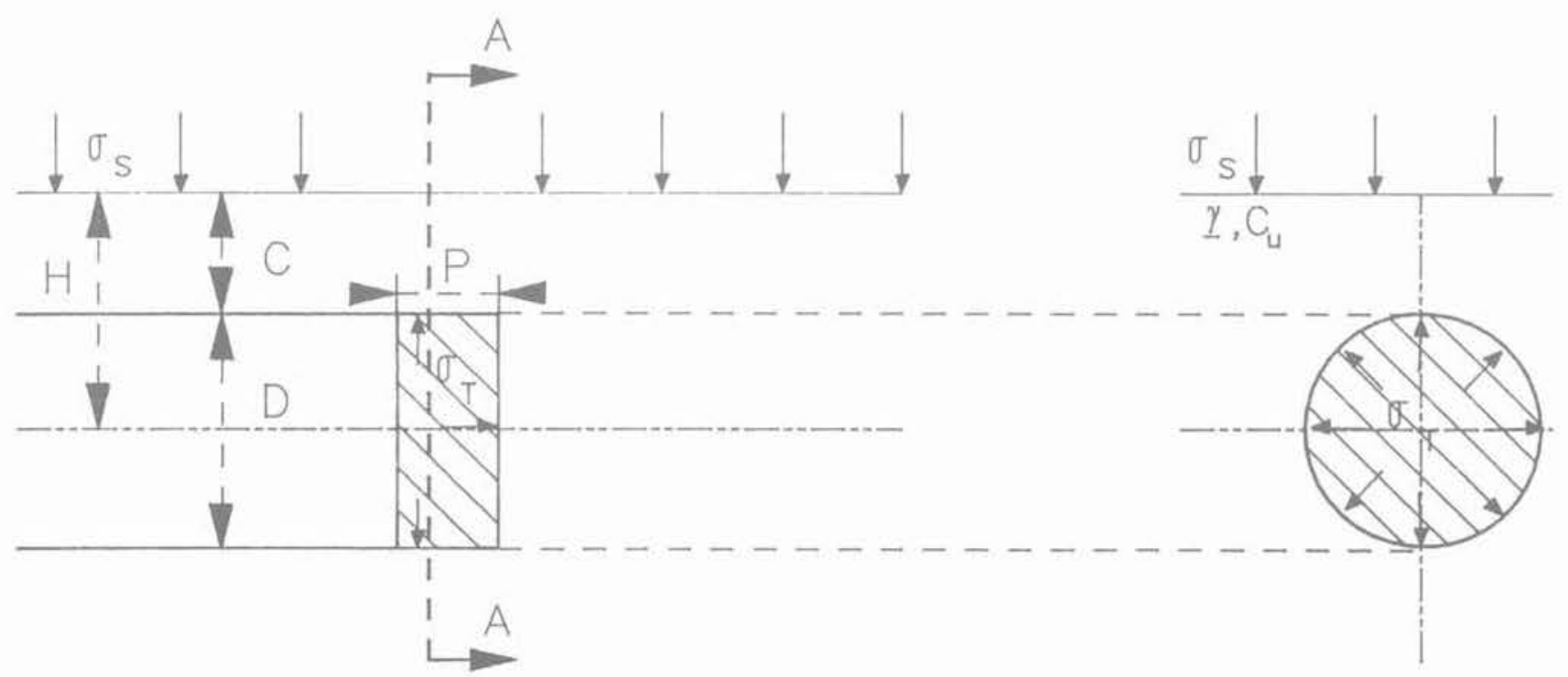

Coupe longitudinale

Coupe $A-A$

Fig. 1. - Schématisation du problème.

Fig. 1. - Simplified geometry 


$$
\mathrm{N}=\frac{\sigma_{\mathrm{s}}+\gamma \mathrm{H}-\sigma_{\mathrm{T}}}{\mathrm{C}_{\mathrm{u}}}
$$

Comme on l'a annoncé, on se propose à présent de construire et de tester plusieurs mécanismes de rupture virtuels afin d'affiner les approches par l'extérieur du domaine de stabilité. Pour chaque mécanisme, une condition nécessaire de stabilité est obtenue en écrivant que la puissance $\mathcal{P}_{\text {ext }}$ des efforts extérieurs dans le mécanisme considéré ne peut excéder la puissance résistante maximale $\mathrm{P}_{\max }^{\text {res }}$ correspondante:

$$
P_{\text {ext }} \leq P_{\max }^{\text {res }}
$$

La quantité $\mathrm{P}_{\max }^{\text {res }}$ est une fonctionnelle du mécanisme virtuel envisagé. Elle ne dépend que du choix de ce dernier et des capacités de résistance du matériau. Elle représente physiquement la puissance maximale que le massif est en mesure de développer dans le mécanisme envisagé, du fait de ses capacités de résistance. L'expression mathématique de cette fonctionnelle est rappelée en annexe.

Pour que la majoration (2) soit non triviale, il est nécessaire que cette puissance $\mathrm{P}_{\max }^{\mathrm{max}}$ soit bornée. Pour un sol purement cohérent (caractérisé par un critère de TRESCA), on montre (SALENÇON, 1983) que ces mécanismes doivent posséder les propriétés suivantes :

1. les éventuelles discontinuités de vitesses doivent être tangentielles :

2. la trace du tenseur taux de déformation doit être nulle en tout point.

\section{MÉCANISMES CONSIDÉRÉS ET RÉSULTATS}

\subsection{Mécanismes de rupture globale, i.e. débouchant en surface}

Le mécanisme proposé par DAVIS et al. (1980) est reproduit sur la figure 2. Il s'agit de deux blocs (1) et (2) cylindriques, de sections elliptiques, animés de mouvements rigidifiants en translation. Les vitesses
$\mathrm{U}_{1}$ du bloc (1) et $\mathrm{U}_{2}$ du bloc (2) sont ajustées de manière à assurer la nullité de la composante normale de la discontinuité de vitesse entre les deux blocs (condition (1) du paragraphe 2).

Ce mécanisme, noté M01, dépend de trois paramètres géométriques: l'angle $\alpha$ entre la verticale et la surface de discontinuité interblocs, langle $\beta$ entre la verticale et laxe du cylindre (2), l'angle $\delta$ entre la surface de discontinuité et l'axe du cylindre (1). L'optimisation du choix de $\alpha, \beta, \delta$ conduit à la condition nécessaire de stabilité la plus restrictive que puisse livrer M01. Elle s'exprime sous la forme d'un majorant du facteur de charge $\mathrm{N}$ défini dans la partie 2 .

Dans le but d'améliorer cette approche par l'extérieur, 3 familles de mécanismes, obtenus en combinant des mouvements virtuels de blocs cylindriques, toriques ou coniques ont été envisagés. Leurs caractéristiques géométriques sont décrites sur les figure 3 à 6 .

Le mécanisme M02 (fig. 3) se compose de trois blocs en mouvement : un cylindre vertical de section droite elliptique en translation (bloc (1)), prolongé par un cylindre en translation (bloc (2)) et un tore (bloc (3)). Dans chaque section droite du bloc (3), la vitesse est constante et perpendiculaire à la section, ce qui signifie que le tore est déformé en distorsion pure. Ce mécanisme dépend de deux paramètres : l'ouverture $\alpha$ du tore et l'angle $\beta$ par rapport à l'horizontale de l'interface entre les blocs (1) et (2).

Le mécanisme M03 (fig. 4) constitue une variante de M02, dans laquelle le bloc vertical (1) est remplacé par deux cylindres de sections droites elliptiques. L'axe de chacun des deux cylindres est perpendiculaire à la surface de contact avec le bloc suivant ; par conséquent ce mécanisme est entièrement défini par les données des deux mêmes paramètres (les angles $\alpha$ et $\beta$ ) que $\mathrm{M} 02$.

Le mécanisme M04 (fig. 5) est construit à partir du mouvement d'un volume tronconique de demi-angle au sommet $\alpha$. L'axe du cône (bloc (2)) est incliné d'un angle $\frac{\pi}{2}-\beta_{2}$ par rapport à la verticale. Le bloc tronconique est prolongé jusqu'à la surface du

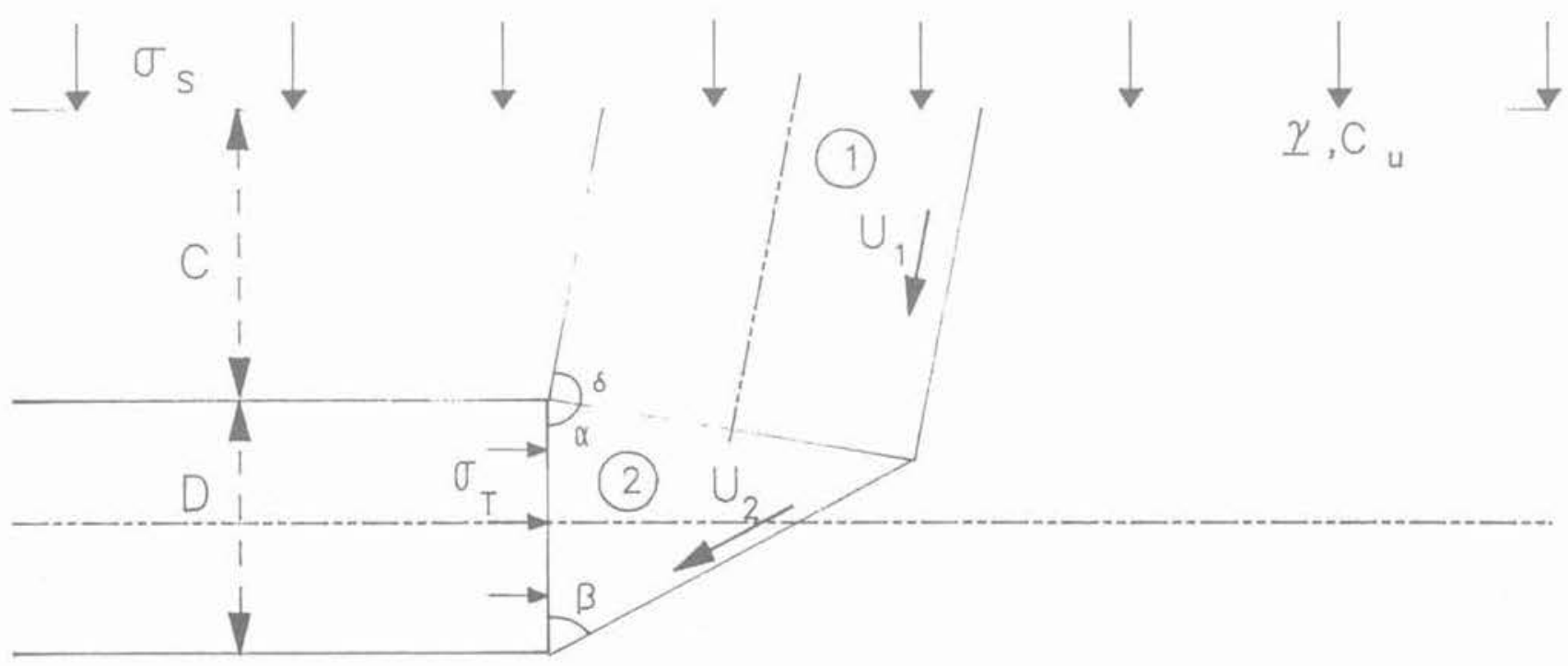

Fig. 2. - Mécanisme M01. - Fig. 2. - Failure mechanism M01. 


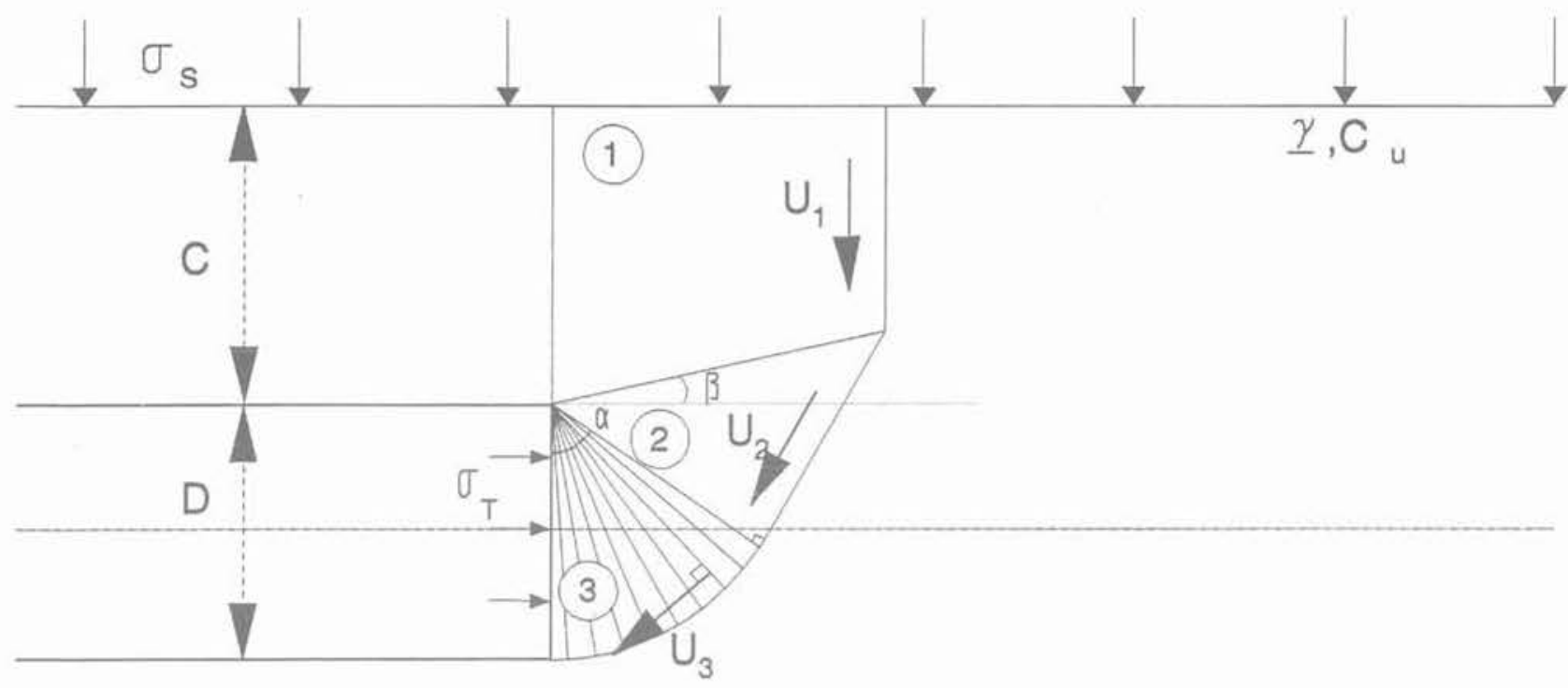

Fig. 3. - Mécanisme MO2. - Fig. 3. - Failure mechanism MO2

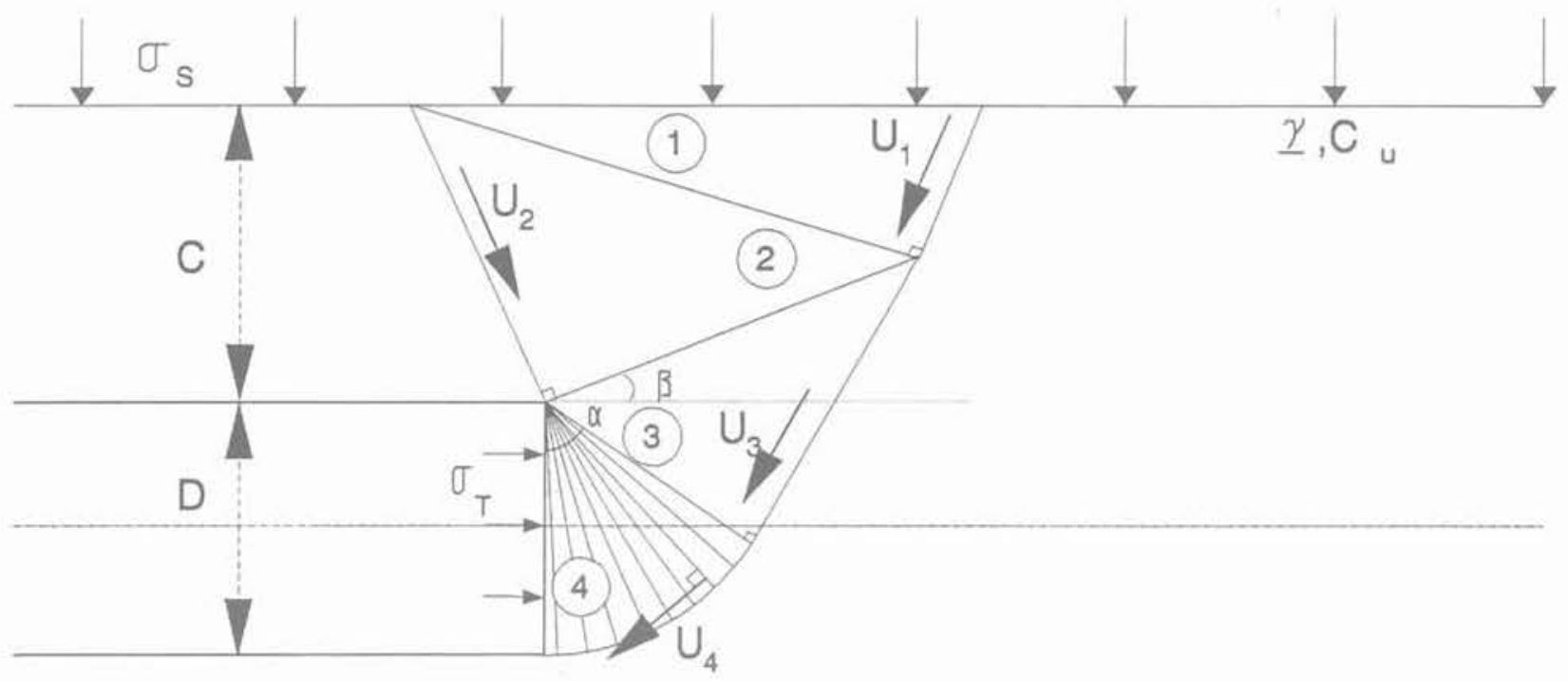

Fig. 4. - Mécanisme M03. - Fig 4 - Failure mechanism M03.

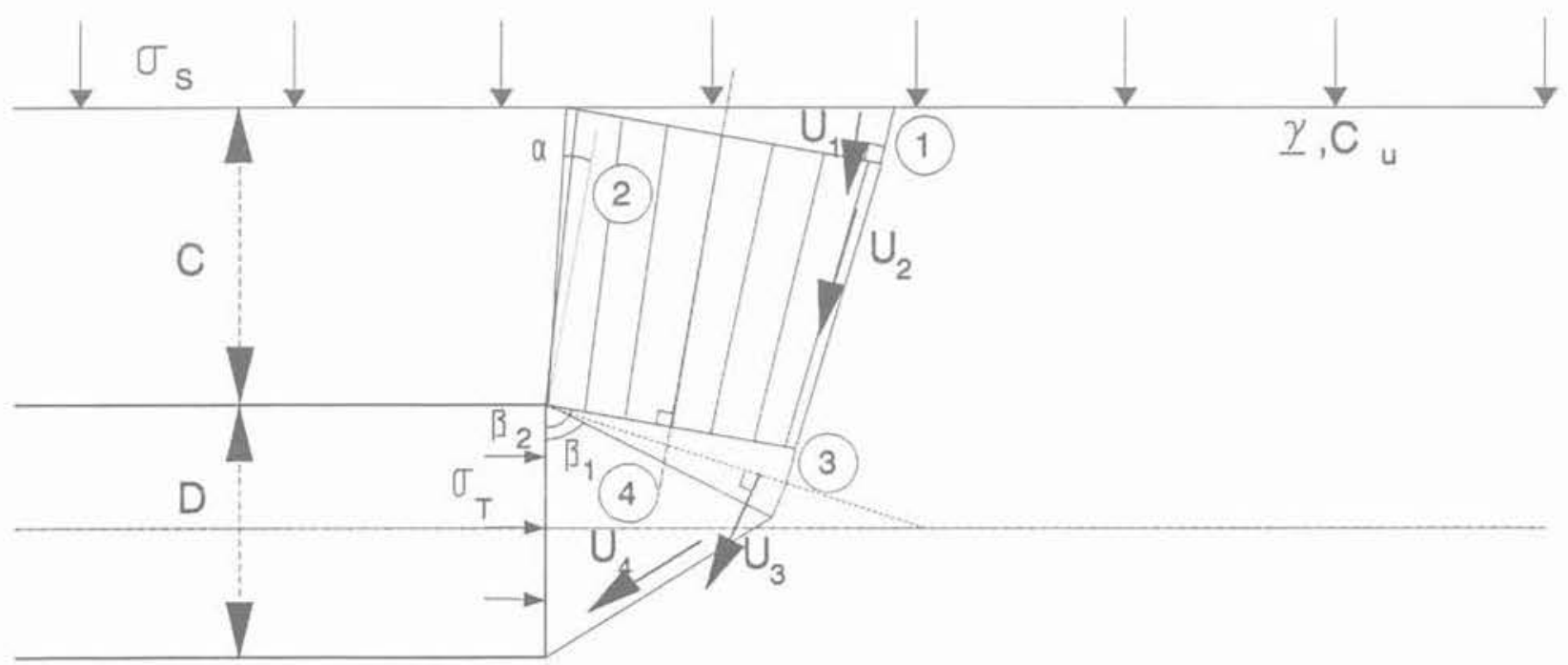

Fig. 5. - Mécanisme MO4. - Fig. 5. - Failure mechanism M04. 


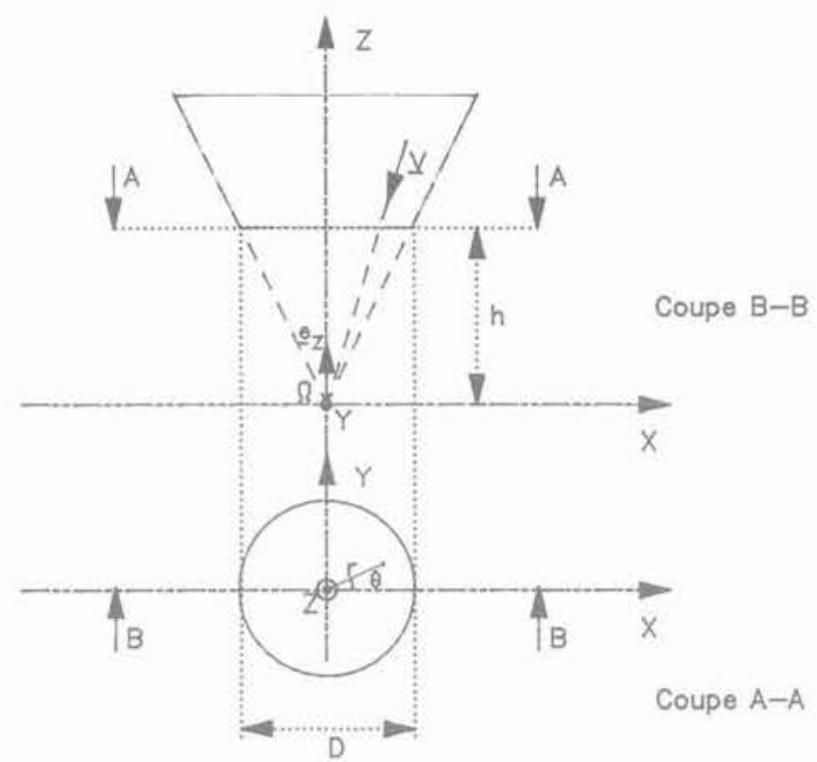

Fig. 6. - Cinématique de bloc conique considéré dans le mécanisme M04.

Fig. 6. - Kinematics of the conical failure mechanism considered in $\mathrm{MO} 04$

sol par un cylindre de section elliptique (bloc (1)) et relié au front de taille du tunnel, par l'intermédiaire d'un tore en distorsion (bloc (3)) et d'un cylindre de section elliptique (bloc (4)). L'ouverture du tore est égale à $\beta_{2}-\beta_{1}$. La cinématique choisie pour le bloc tronconique est décrite sur la figure 6 . $\Omega$ désignant le sommet du cône et $\Omega$ u son axe (vecteur unitaire directeur $e_{u}$ ), le vecteur-vitesse en un point $M$ du tronc de cône est donné par la relation :

$$
\underline{V}(\mathrm{M})=-U h^{2} \frac{\underline{\Omega M}}{\left(\Omega \mathrm{M} \cdot \underline{e}_{u}\right)^{3}}
$$

où U est la norme de la vitesse à l'intérieur du tore. Un tel choix assure la nullité de la composante normale de la vitesse sur les faces latérales du cône. Ce champ de vitesses virtuel correspond à une distorsion du bloc tronconique sans déformation volumique. Le mécanisme M04 dépend de trois paramètres : $\alpha, \beta_{1}$ et $\beta_{2}$.

Pour chaque mécanisme, une condition nécessaire de stabilité est obtenue en écrivant la relation (2), pour les valeurs des puissances $P_{\text {ex }}$ et $P_{\max }^{\text {res }}$ correspondantes. La condition la plus restrictive associée à un mécanisme donné est déterminée après optimisation sur les paramètres cinématiques mis en jeu.

Tous les mécanismes examinés permettent de déterminer un majorant de la valeur extrême, notée $\mathrm{N}^{+}$. du facteur de charge. Ce majorant dépend du rapport $C / D$. Les résultats sont rassemblés sur la figure 7 , pour des valeurs de $C / D$ variant entre 0 et 5 . Les variations en fonction de $\mathrm{C} / \mathrm{D}$ du plus petit majorant,

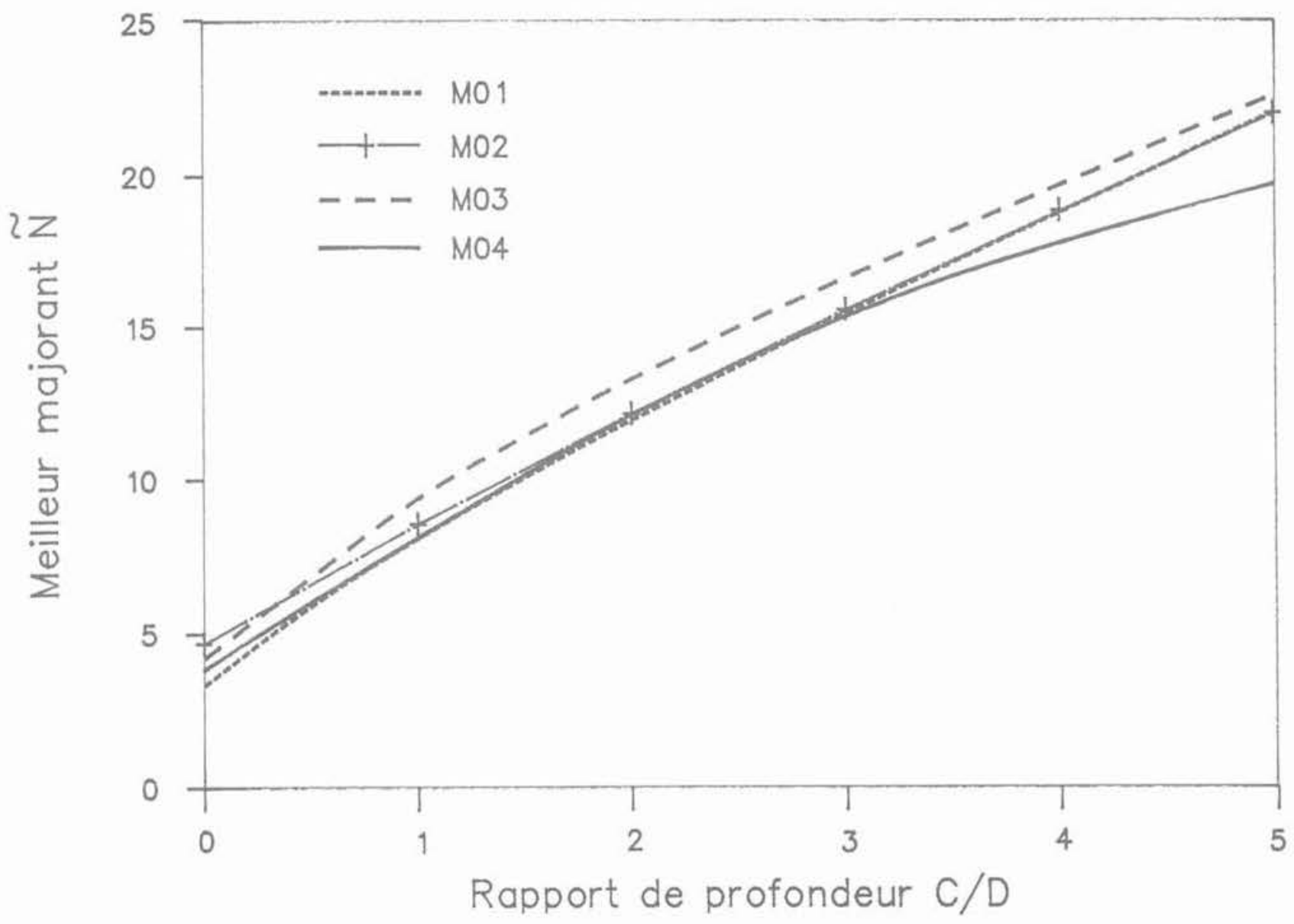

Fig. 7. - Comparaison des valeurs de $\tilde{N}$ obtenues en rupture globale.

Fig. 7. - Comparison between the $\tilde{N}$ values obtained for global failure. 
noté $\widetilde{\mathrm{N}}$ dans la suite, sont données par l'enveloppe inférieure des courbes représentant chacun des mécanismes.

Pour des tunnels creusés à faible profondeur $(C / D$ $<3,0$ ), on constate que le meilleur majorant $\widetilde{\mathrm{N}}$ correspondant pratiquement au mécanisme M01 proposé par DAVIS et al. (1980).

Le meilleur majorant $\tilde{\mathrm{N}}$ est rigoureusement donné par M01 pour $C / D=0,0$. Pour les autres valeurs de $C / D$, des variantes du mécanisme M01, obtenues par adjonction d'un coin torique en distorsion entre les deux blocs cylindriques (mécanisme M04 avec $\alpha=0$ ) permettent d'améliorer l'estimation du facteur de charge limite, mais pas de manière significative. En revanche, aux grandes profondeurs $(C / D>3,0)$, le mécanisme M04 conduit à des majorants du facteur de charge extrême $\mathrm{N}^{+}$sensiblement meilleurs que ceux fournis par DAVIS et al. (1980).

\subsection{Mécanismes de rupture locale, i.e. ne débouchant pas en surface}

Pour étudier la stabilité locale du front de taille d'un tunnel en milieu cohérent, le mécanisme imaginé par DAVIS et al. (1980) (mécanisme M05, fig. 8) correspond au mouvement de rotation d'une calotte sphérique centrée en un point $\Omega$ de l'axe du tunnel (défini par l'angle d'ouverture $\alpha$ ) et s'appuyant sur la circonférence du front de taille.

En optimisant le choix de $\alpha$, la condition de stabilité locale la plus restrictive se met sous la forme d'un majorant de la valeur extrême $\left(\gamma \mathrm{D} / \mathrm{C}_{\mathrm{u}}\right)^{+}$du rapport $\gamma \mathrm{D} / \mathrm{C}_{u}$ :

$$
\left(\gamma \mathrm{D} / \mathrm{C}_{\mathrm{u}}\right)^{+} \leq 10,96
$$

qui constitue la meilleure borne donnée par la littérature.

\section{4. ÉTUDE DE L'INFLUENCE DU CRITÈRE DE RÉSISTANCE DU TERRAIN}

\subsection{Position du problème}

L'étude qui vient d'être présentée était destinée à améliorer l'approche par l'extérieur du domaine des chargements potentiellement supportables pour le problème de la stabilité du front de taille en terrain cohérent. Malgré la diversité des mécanismes envisagés. lamélioration obtenue n'est, en général, pas significative. Les raisons de l'écart entre la frontière livrée par l'approche cinématique et les résultats expérimentaux restent donc à clarifier.

En modélisant les capacités de résistance du matériau par le critère de TRESCA :

$$
f(\underline{\underline{\sigma}})=\sup _{i}\left(\sigma_{i}-\sigma_{j}\right)-2 C_{u} \leq 0
$$

on attribue au sol une résistance à la traction qui n'est pas mobilisable (au moins intégralement) en réalité. On peut donc se demander si l'écart entre l'approche cinématique et les résultats expérimentaux peut être expliqué par une surestimation du domaine des contraintes admissibles du matériau. En d'autres termes, est-il possible d'obtenir un meilleur accord entre les résultats expérimentaux et les résultats d'une étude théorique de la stabilité (approches statique et cinématique), en modélisant les capacités de résistance du matériau par un critère de TRESCA sans résistance à la traction? Rappelons que la forme mathématique de ce critère est la suivante :

$$
\mathrm{f}^{\prime}(\underline{\underline{\sigma}})=\sup _{i, j}\left(\sigma_{i}-\sigma_{i}-2 \mathrm{C}_{\mathrm{u}} ; \sigma_{\mathrm{i}}\right) \leq 0
$$

Voyons, sur le cas de l'approche cinématique, comment le passage du critère (5) au critère (6) peut a priori conduire à des conditions de stabilité plus restrictives. Etant donné un champ de vitesses virtuelles $\mathrm{U}$, le théorème cinématique fournit classiquement une condition nécessaire de stabilité sous la forme

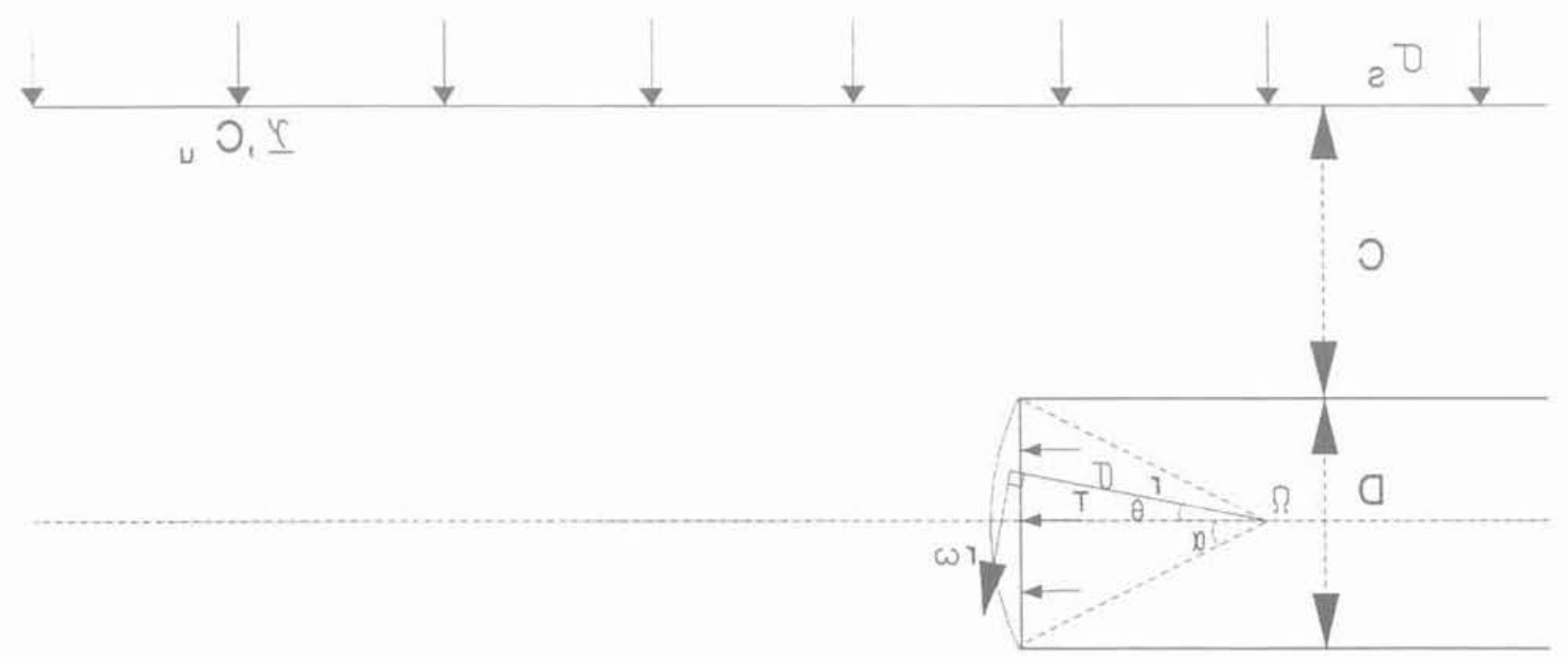

Fig. 8. - Mécanisme MO5.

Fig. 8. - Failure mechanism MO5 
énoncée en (2). Comme on l'a vu $\mathscr{P}_{\text {ext }}(\underline{U}, Q)$ représente la puissance du chargement examiné, défini par le vecteur $Q$ des paramètres de chargement, dans le champ de vitesses $\mathrm{U}$; elle est indépendante des propriétés du matériau. L'expression de la puissance résistante maximale est donnée en (A1). Cette quantité est indépendante du chargement. D'une manière évidente, les fonctions $\pi$ définies en (A2) sont des "fonctions croissantes du domaine des contraintes admissibles". Plus précisément, considérant deux matériaux possédant les domaines de contraintes admissible $G^{1}$ et $G^{2}$, on a manifestement, en vertu de la définition donnée en (A2) :

$$
\mathrm{G}^{1} \subset \mathrm{G}^{2} \Rightarrow \pi^{1} \leq \pi^{2}
$$

En d'autres termes, la puissance résistante maximale est une fonction croissante (au sens large) du domaine des contraintes admissibles. C'est pourquoi il est naturel d'espérer que les conditions de stabilité résultant de l'approche cinématique soient plus restrictives (et donc plus proches des résultats expérimentaux) si l'on remplace le critère $f$ avec résistance à la traction par le critère $f^{\prime}$ sans résistance à la traction.

Notons cependant avant tout calcul qu'il ne s'agit pas là d'une règle générale. Pour le problème classique de la stabilité d'une excavation verticale de hauteur $\mathrm{H}$ réalisée dans un massif constitué d'un matériau pesant (poids volumique $\gamma$ ) et cohérent (cohésion $\mathrm{C}_{1}$ ), on montre (voir par exemple SALENCON. 1983) que les conditions de stabilité obtenues par la cinématique sont beaucoup plus restrictives en l'absence de résistance à la traction :

$$
\begin{array}{ll}
\gamma \mathrm{H} / \mathrm{C}_{\mathrm{u}} \leq 3,83 & \text { dans le cas du critère } \mathrm{f} \\
\gamma \mathrm{H} / \mathrm{C}_{\mathrm{u}} \leq 2 & \text { dans le cas du critère } \mathrm{f}
\end{array}
$$

En revanche, pour une fondation superficielle soumise à une charge verticale centrée, on peut montrer qu'un défaut de résistance à la traction ne modifie pas la capacité portante. Mais ce résultat devient inexact si la fondation est soumise à une charge inclinée (PEC. KER et SALENCON, 1991, 1992).

Ainsi, la manifestation d'un défaut de résistance à la traction n'est pas systématique : elle dépend du cas de charge considéré. Sans reprendre d'une manière détaillée l'approche cinématique dans le cadre du critère $f$, on se propose néanmoins maintenant, sur l'exemple de la stabilité "locale * du front de taille. de mettre en évidence des différences substantielles selon que l'on adopte l'un ou l'autre des critères (5) ou (6). On se limitera dans la suite à un mécanisme de bloc. Il convient donc tout d'abord de rappeler lexpression de la fonction $\pi(n, U)$ du matériau sans résistance à la traction défini par (6).

\subsection{Calcul de la puissance résistante maximale pour le matériau sans résistance à la traction}

La figure 9 représente la courbe intrinsèque du matériau cohérent sans résistance à la traction (convention des contraintes positives en traction). Elle délimite dans le plan de Mohr $\left(\sigma_{n}, \tau\right)$ l'ensemble $\mathscr{I}$ des vecteurs-contraintes $\mathrm{T}=\sigma_{\mathrm{n}} \mathrm{n}+\tau \mathrm{t}$ admissibles. On a donc :

$$
\pi(\underline{n}, \underline{U})=\sup (\underline{T} \cdot \underline{U}, \underline{I} \in \text { I) }
$$

Si le produit scalaire $\mathrm{U}$. $\mathrm{n}$ est négatif, la quantité $\underline{\mathrm{U}}$. I n'est pas bornée lorsque $\mathrm{I}$ décrit $\mathfrak{I}$. Il suffit d'observer que le vecteur-contrainte $\mathrm{T}=\alpha \mathrm{n}$ est élément de $\mathfrak{g}$ pour toute valeur négative $\alpha, \bar{O}_{n}$ a donc :

$$
\underline{\mathrm{U}} \cdot \underline{\mathrm{n}} \leq 0 \Rightarrow \pi(\underline{\mathrm{n}}, \underline{\mathrm{U}})=+\infty
$$

Dans la situation représentée à la figure 9 , où $\mathrm{U} . \mathrm{n} \geq 0$, il est facile de voir que le vecteur $T$ $=$ OT qui réalise le maximum du produit scalaire $\underline{\mathrm{U}} . \underline{\mathrm{T}}$ est tel que $\mathrm{CT}$ soit parallèle à $\underline{\mathrm{U}}$. On a donc :

$$
\begin{aligned}
\underline{\mathrm{U}} \cdot \underline{\mathrm{n}} \geq & 0 \Rightarrow \pi(\underline{\mathrm{n}}, \underline{\mathrm{U}})=\underline{\mathrm{U}} \cdot(\underline{\mathrm{OC}}+\underline{\mathrm{CT}}) \\
& =\mathrm{C}_{\mathrm{u}}(\|\underline{\mathrm{U}}\|-\underline{\mathrm{U}} \cdot \underline{\mathrm{n}})
\end{aligned}
$$

D'une manière générale, les mécanismes pertinents pour l'approche cinématique sont, parmi tous les champs de vitesses virtuelles, ceux pour lesquels la puissance résistante maximale est finie. En effet. cette restriction est évidemment nécessaire pour que la condition de stabilité (2) soit non triviale. Pour le matériau décrit par $\mathrm{f}$, on sait que les discontinuités des mécanismes pertinents sont assujetties à être tangentielles. Les équations (9) et (10) indiquent que cette restriction est partiellement levée dans le cas du matériau cohérent sans résistance à la traction pour lequel la discontinuité de vitesse est "pertinente " au sens précédent dès lors qu'elle correspond à un décollement $([\underline{U})] \cdot \underline{n} \geq 0$ ). Pour les applications pratiques, cela implique, comme on pouvait s'y attendre, que les conditions de stabilité obtenues par voie cinématique pour le critère $f$ subsistent pour le critère $f^{\prime}$.

Cependant, la classe des mécanismes pertinents se trouvant élargie, de nouveaux mécanismes susceptibles de conduire à des conditions de stabilité plus restrictives doivent maintenant être examinés.

\subsection{Stabilité locale du front de taille}

En matière de stabilité locale du front de taille, le meilleur résultat disponible est celui de DAVIS et al. (1980), qui a été décrit au paragraphe (3.2.). On rappelle qu'il est obtenu en explorant un mode de rupture par rotation d'une calotte sphérique centrée sur laxe du tunnel et s'appuyant sur le contour du front de taille.

On considère un arc de tore II d'axe perpendiculaire au plan de symétrie Oxy de l'ouvrage, passant par la clé $\mathrm{C}$ du tunnel dans le plan du front de taille (voir mécanisme M06. fig. 10). Le tore est engendré par la rotation autour de l'axe $\mathrm{CZ}$ d'un disque de rayon $\mathrm{R}$; le centre du disque décrit autour de $\mathrm{CZ}$ un arc de cercle de rayon $\mathrm{R}$ et d'ouverture $\alpha$. On fait l'hypothèse que l'arc de tore ne débouche pas à la surface du massif. On envisage le mode de rupture par rotation de l'arc de tore autour de son axe à la vitesse $\underline{\omega}=-\omega \underline{e}_{2}(\omega>0)$ :

$$
\underline{\mathrm{U}}=-\underline{\omega e}_{z} \wedge \underline{\mathrm{CM}}
$$




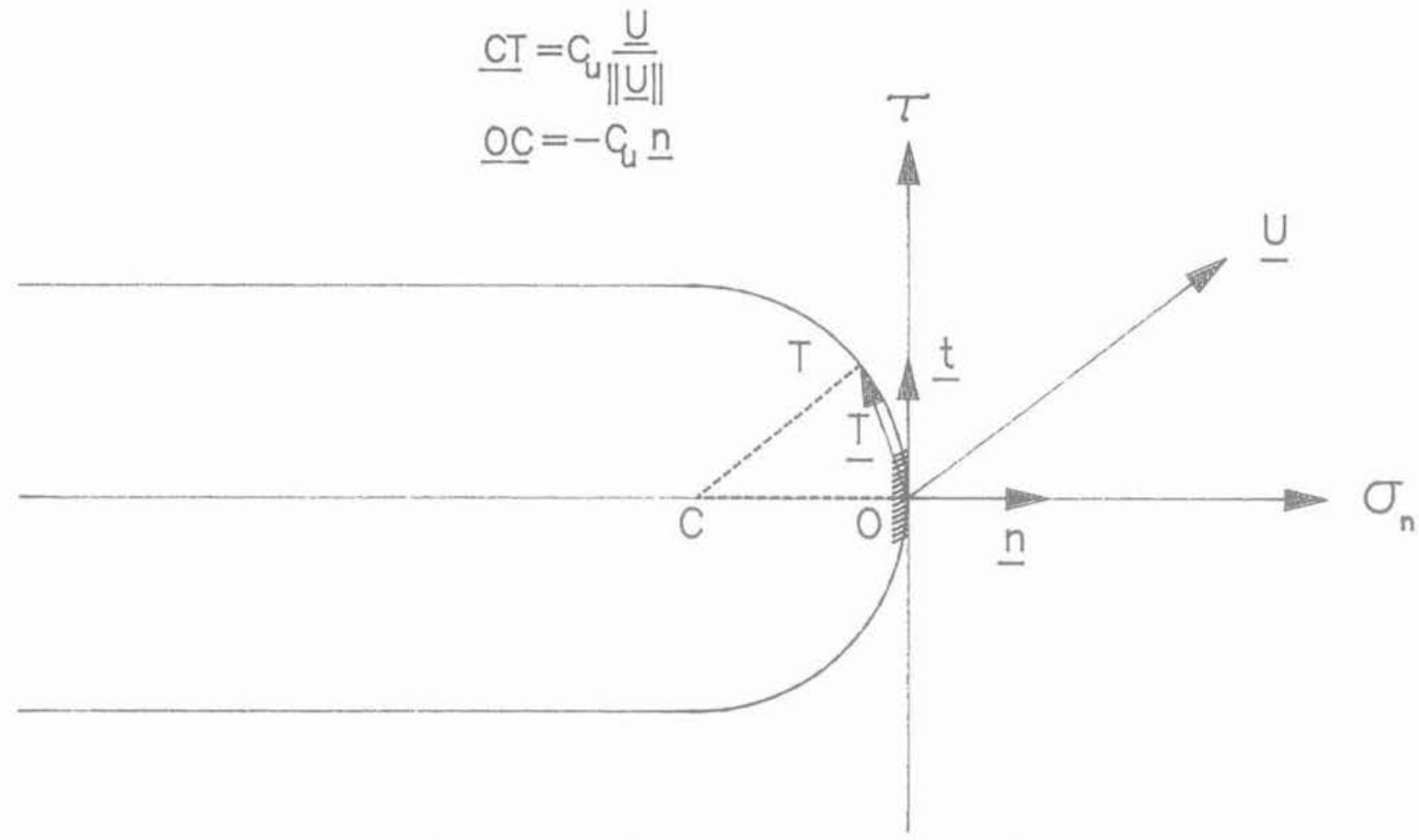

Fig. 9. - Critère de Tresca sans résistance à la traction.

Fig. 9. - Tresca criterion with tension cut-off.
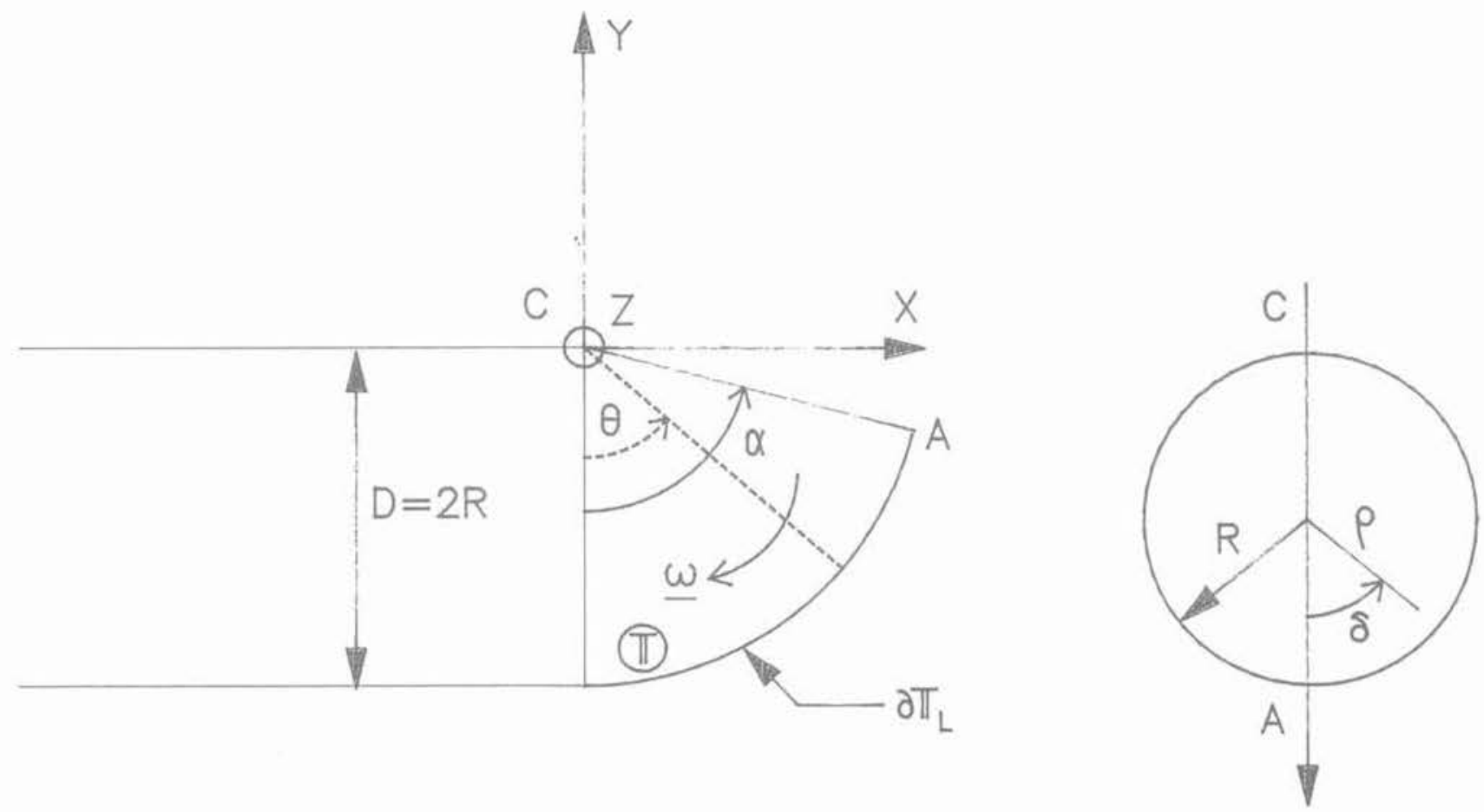

Fig. 10. - Mécanisme MO6.

Fig. 10, - Failure mechanism MO6.

On utilise le paramétrage $\rho, \delta, \theta$ défini à la figure 10 . Le mécanisme étudié présente une discontinuité de vitesse purement tangentielle sur la face latérale du tore $\partial \Pi_{\mathrm{L}}$. Par ailleurs, il présente une discontinuité de vitesses purement normale (de décollement) au niveau du disque contenant le segment $\mathrm{CA}$, formant 
langle $\theta=\alpha$ avec la direction verticale descendante. En vertu de la formule (10), il est facile de voir que seule la face latérale $\partial \Pi_{\mathrm{L}}$ contribue à la puissance résistante maximale dans le mécanisme considéré

$$
\begin{aligned}
& \mathrm{P}_{\max }^{\text {mes }}=\int_{\partial \pi_{\mathrm{L}}} \mathrm{C}_{\mathrm{u}}\|\underline{\mathrm{U}}\| \mathrm{dS} \\
& =\int_{\partial \pi_{\mathrm{L}}} \mathrm{C}_{\mathrm{u}} \omega\|\underline{\mathrm{CM}}\| \mathrm{dS}
\end{aligned}
$$

soit en utilisant le paramétrage de $\partial \mathrm{T}_{\mathrm{L}}$ en fonction de $\theta$ et $\delta$ :

$$
\begin{aligned}
\mathrm{P}_{\max }^{\mathrm{res}}=\mathrm{C}_{\mathrm{u}} & \omega \mathrm{R}^{3} \int_{0}^{\alpha} \mathrm{d} \theta \int_{0}^{2 \pi} \mathrm{d} \delta(1+\cos \delta)^{2} \\
& =3 \mathrm{C}_{\mathrm{u}} \omega \pi \mathrm{R}^{3} \alpha
\end{aligned}
$$

Dans le mécanisme étudié, les forces de pesanteur sont motrices et la pression de bouclier est résistante. La puissance des forces de pesanteur est la suivante:

$$
P_{\gamma}=\int_{I I} \underline{U} \cdot\left(-\underline{\varepsilon_{y}}\right) d V
$$

soit explicitement, en tenant compte de (11):

$$
\begin{gathered}
\rho_{\gamma}=\gamma \omega \int_{0}^{\alpha} \sin \theta \mathrm{d} \theta \int_{0}^{\mathrm{R}} \rho \mathrm{d} \rho \int_{0}^{2 \pi} \mathrm{d} \delta(\rho \cos \delta+\mathrm{R})^{2} \\
=\frac{5}{4} \gamma \omega(1-\cos \alpha) \pi \mathrm{R}^{4}
\end{gathered}
$$

La puissance de la pression de bouclier s'exerçant sur le front de taille a l'expression suivante:

$$
P_{\mathrm{T}}=\int_{\xi} \underline{U} \cdot\left(-\sigma_{\mathrm{T}} \underline{e}_{x}\right) \mathrm{dS}
$$

soit explicitement

$$
\begin{gathered}
P_{\mathrm{T}}=-\sigma_{\mathrm{T}} \omega \int_{0}^{\mathrm{R}} \rho \mathrm{d} \rho \int_{0}^{2 \pi}(\rho \cos \delta+\mathrm{R}) \mathrm{d} \delta \\
=-\sigma_{\mathrm{T}} \omega \pi \mathrm{R}^{3}
\end{gathered}
$$

Pour toute valeur de $\alpha \in[0, \pi]$, le théorème cinématique fournit une condition de stabilité sous la forme :

$$
\frac{5}{8}(1-\cos \alpha)\left(\gamma \mathrm{D} / \mathrm{C}_{\mathrm{u}}\right)-\left(\sigma_{\mathrm{T}} / \mathrm{C}_{\mathrm{u}}\right) \leq 3 \alpha
$$

Le mécanisme de calotte sphérique de DAVIS et al. (1980) présente l'avantage de ne pas faire "travailler « la pression de bouclier. Il sera donc meilleur pour les valeurs élevées de celle-ci. En revanche, en labsence de pression au front de taille, le mécanisme de tore fournit, en vertu de (18), la condition :

$$
\gamma \mathrm{D} / \mathrm{C}_{\mathrm{u}} \leq 24 / 5 \inf _{0<\alpha \leq \pi} \frac{\alpha}{(1-\cos \alpha)}=6,62
$$

La valeur de langle $\alpha$ correspondant à l'optimum vaut $0,74 \pi$.

En comparant la condition (19) à (4), il apparaît clairement qu'un défaut de résistance en traction doit impérativement être pris en compte dans l'analyse du risque d'instabilité locale. Pour les faibles valeurs de la pression de bouclier, un mécanisme de rupture du type qui vient d'être étudié peut s'initier. Par ailleurs, la condition (19) a pu être obtenue très simplement en explorant une classe de mécanismes dépendant d'un unique paramètre. L'objectif était de mettre en évidence les conséquences d'un défaut de résistance en traction. Cependant, il est vraisemblable que la condition (19) pourrait être elle-même substantiellement améliorée par une étude plus complète.

\subsection{Approche statique}

On considère deux critères de rupture $f_{1}$ et $f_{2}$ correspondant en tout point $\mathrm{x}$ du domaine matériel $\Omega$ à des domaines de contraintes admissibles ordonnés au sens de l'inclusion selon $G^{2}(x) \subset G^{1}(x)$. Les approches par l'intérieur du domaine $\mathrm{K}$ des chargements potentiellement supportables effectuées par la méthode statique sont évidemment ordonnées dans le même sens. Il convient donc d'examiner si le passage à un critère sans résistance à la traction fait " chuter " l'estimation par défaut du domaine $\mathrm{K}$ correspondant au critère non tronqué en traction. Trois champs de contraintes permettent classiquement de construire celle-ci. Ils ont été décrits par DAVIS et al. (1980). Le premier champ de contrainte noté $\underline{\underline{g}}^{(1)}$ est statiquement admissible dans le cas du matériau pesant. Les deux autres champs de contraintes sont statiquement admissibles dans le cas du matériau non pesant. Ils sont construits en exploitant respectivement une symétrie sphérique (champ $\underline{\sigma}^{(2)}$ ) ou cylindrique partielle du problème. On montre cependant que le champ à symétrie sphérique fournit une approche statique meilleure dans le domaine $C / D \geq 0,83$ auquel on se limitera.

On établit ci-après que l'estimation par défaut de $\mathrm{K}$ obtenue classiquement pour le critère $f$ au moyen des champs $\underline{g}^{(1)}$ et $\underline{g}^{(2)}$ reste valable pour le matériau sans résistance à la traction dans le domaine des valeurs usuelles de $\sigma_{\mathrm{S}} / \mathrm{C}_{\mathrm{u}}$ et $\sigma_{\mathrm{T}} / \mathrm{C}_{\mathrm{u}}$.

\subsubsection{Etude du cas « pesant »}

Le champ de contraintes $\underline{\underline{\sigma}}{ }^{(1)}$ est en équilibre avec une pression $\sigma_{\mathrm{T}}$ dans le tunnel, une pression $\sigma_{5}$ en surface et les forces de pesanteur agissant sur le sol de poids volumique $\gamma$. Dans les axes de la figure 11 , il est défini de la manière suivante:

- dans le cylindre en pointillés dans le prolongement du tunnel :

$$
\begin{aligned}
& \underline{\underline{\sigma}}^{(1)}=-\sigma_{\mathrm{T}} \underline{e}_{z} \otimes \underline{e}_{z}+\left(\gamma(y-H)-\sigma_{s}\right) \\
& \left\{\underline{e}_{x} \otimes \underline{e}_{\mathrm{x}}+\underline{e}_{y} \otimes \underline{e}_{y}\right\}
\end{aligned}
$$

- dans le reste du massif, le champ de contraintes est sphérique :

$$
\left.\underline{\underline{g}}^{(1)}=(\gamma(y-H))-\sigma_{s}\right) \underline{\underline{1}}
$$




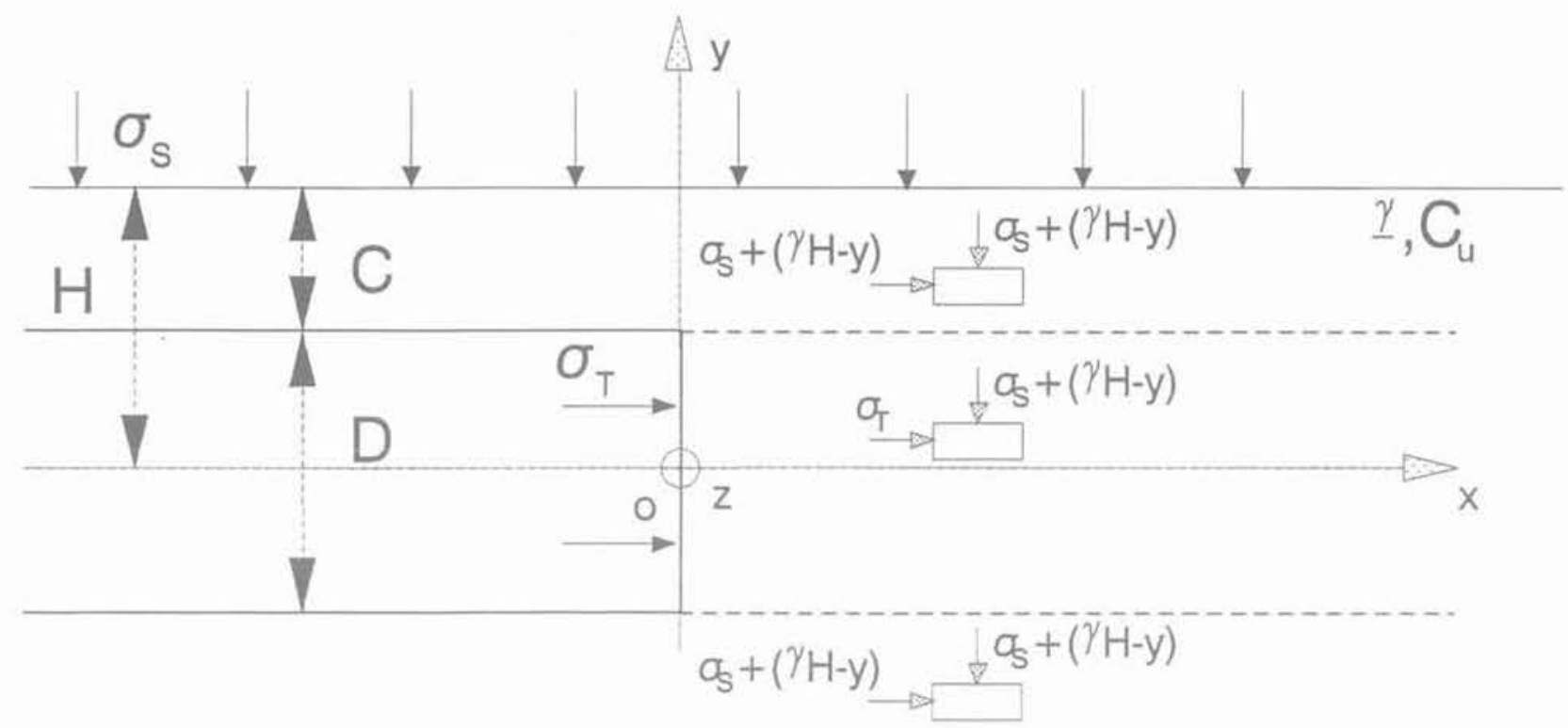

Fig. 11. - Champ de contrainte $\sigma^{(1)}$.

Fig. 11. - Stress field $\sigma^{(1)}$

Les quantités $\sigma_{5}$ et $\sigma_{\mathrm{T}}$ étant évidemment positives, toutes les contraintes principales sont des contraintes de compression. Il en résulte que les critères $f$ et $f$ prennent la même valeur en $\underline{g}^{(1)}$. En d'autres termes, on obtient au moyen de $\sigma^{(1)}$ la même approche par l'intérieur de $\mathrm{K}$, que le matériau résiste ou non à la traction. On rappelle qu'elle est définie par les conditions suivantes :

$$
\mathrm{N}+\frac{\gamma \mathrm{D}}{2 \mathrm{C}_{\mathrm{u}}} \leq 2 \quad-\mathrm{N}+\frac{\gamma \mathrm{D}}{2 \mathrm{C}_{\mathrm{u}}} \leq 2
$$

où l'on a posé $\mathrm{N}=\left(\sigma_{\mathrm{s}}-\sigma_{\mathrm{T}}+\gamma \mathrm{H}\right) / \mathrm{C}_{\mathrm{u}}$.

\subsubsection{Etude du cas « non pesant »}

Dans le cas particulier du matériau non pesant, les pressions $\sigma_{\mathrm{T}}$ et $\sigma_{\mathrm{s}}$ constituent les deux paramètres de chargement. On adopte le système de coordonnées sphériques de centre 0 . On désigne par $\left(M, \underline{e}_{r}, \underline{e}_{\theta}\right.$. $\left.\underline{e}_{\varphi}\right)$ le repère local orthonormé. Le champ $\underline{\underline{g}}^{(2)}$ est défini de la manière suivante (voir figure 12) :

- à lintérieur de la sphère de centre 0 de rayon $R=D / 2$ :

$$
\underline{\underline{\sigma}}^{(2)}=-\sigma_{\mathrm{T}} \underline{\underline{1}}
$$

- à l'extérieur de la sphère de centre 0 de rayon $\mathrm{H}$ :

$$
\underline{\underline{g}}^{(2)}=-\sigma_{\mathrm{s}} \underline{\underline{1}}
$$

- entre ces deux sphères :

$$
\underline{\underline{\sigma}}^{(2)}=f(r) \underline{e}_{r} \otimes \underline{e}_{r}
$$

$\left.+(f(r)-k) \underline{e}_{\theta} \otimes \underline{e}_{\theta}+\underline{e}_{\varphi} \otimes \underline{e}_{\varphi}\right\}$

où l'on a posé :

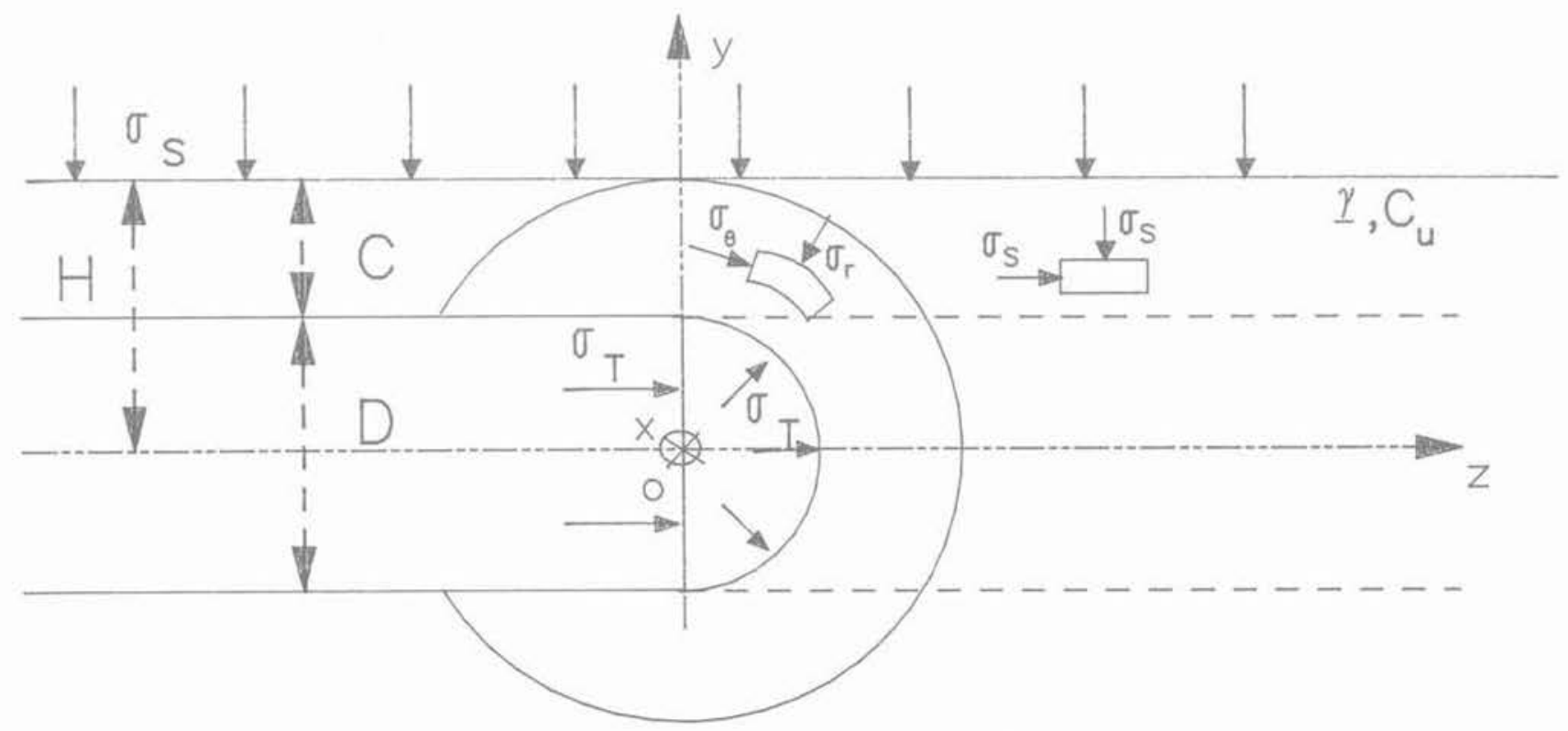

Fig. 12. - Champ de contrainte $\sigma^{(2)}$.

Fig. 12. - Stress field o ${ }^{(2)}$ 


$$
\begin{aligned}
& f(r)=-\left(\sigma_{s}-\sigma_{T}\right) \frac{\ln (2 \mathrm{r} / \mathrm{D})}{\ln (2 \mathrm{H} / \mathrm{D})}-\sigma_{T} \\
& \mathrm{k}=\frac{\sigma_{\mathrm{s}}-\sigma_{\mathrm{T}}}{2 \ln (2 \mathrm{H} / \mathrm{D})}
\end{aligned}
$$

Pour que les contraintes principales de $\underline{\sigma}^{(2)}$ soient toutes de compression, il faut et il suffit que :

$$
\sigma_{\mathrm{T}} / \sigma_{\mathrm{s}} \leq 1+\frac{1}{2 \ln (2 \mathrm{H} / \mathrm{D})}
$$

Sous réserve que cette condition soit vérifiée, une approche par l'intérieur de $\mathrm{K}$ pour le matériau sans résistance à la traction est définie par les conditions :

$$
\left|\sigma_{\mathrm{S}}-\sigma_{\mathrm{T}}\right| / \mathrm{C}_{\mathrm{u}} \leq 4 \ln (2 \mathrm{H} / \mathrm{D})
$$

Dans la pratique, on recherche la valeur minimale de $\sigma_{\mathrm{T}}$ requise pour assurer la stabilité, la pression $\sigma_{\mathrm{s}}$ étant une donnée du problème. On travaille donc en général dans le domaine $\sigma_{\mathrm{s}} \geq \sigma_{\mathrm{T}}$. L'approche par l'intérieur du domaine des chargements potentiellement supportables qui vient d'être rappelée est alors définie par l'unique condition $\left(\sigma_{\mathrm{s}}-\sigma_{\mathrm{T}}\right) / \mathrm{C}_{\mathrm{u}} \leq 4 \ln$ (2H/D). Elle coïncide avec la condition obtenue pour le critère $f$.

\section{CONCLUSION}

Il convient de rappeler que le point de départ de ce travail réside dans le fait que les résultats expérimentaux présentés par DAVIS et al. (1980) semblent indiquer que la frontière du domaine des chargements supportés est voisine de l'approche par l'intérieur par les contraintes,

Les géométries critiques observées sur des essais de rupture en centrifugeuse (SCHOFIELD, 1980) présentent certaines similarités avec le mécanisme de blocs proposé dans l'étude de DAVIS et al. (1980). Toutefois, cette même étude montre des différences assez importantes entre les valeurs des pressions de soutènement critiques observées expérimentalement et celles obtenues à partir d'un calcul basé sur ce mécanisme.

Il était donc naturel de tenter d'affiner l'approche cinématique vis-à-vis des risques de ruptures globale et locale. Plusieurs familles de nouveaux mécanismes de rupture ont été envisagées, à partir de blocs cylindriques, toriques, coniques animés de mouvements rigidifiants ou soumis à des déformations. Malgré leur diversité, les mécanismes considérés ne permettent pas en général d'apporter des améliorations significatives par rapport aux résultats de DAVIS et al. (1980), sauf dans le domaine $\mathrm{C} / \mathrm{D}>3$.

Ce résultat a conduit à réexaminer la modélisation des capacités de résistance de l'argile en condition non drainée, qui sont classiquement représentées par un critère de TRESCA. Celui-ci présente l'inconvénient de surestimer le domaine des contraintes admissibles. dans la mesure où il confère au sol une résistance en traction égale à sa résistance en compression.
L'influence d'un défaut de résistance à la traction a donc été analysée dans le cas particulier d'un mode de rupture locale. Le mécanisme étudié fournit, dans certaines conditions, une amélioration substantielle de la borne supérieure donnée par DAVIS et al. pour l'effondrement local.

Une étude plus poussée de la stabilité du front de taille en terrain cohérent, tenant compte d'un défaut de résistance en traction, serait nécessaire avant de pouvoir établir des conclusions plus générales. Cependant, les premiers résultats obtenus en rupture locale à partir d'un critère de rupture tronqué en traction sont encourageants. Cette idée permet d'ores et déjà d'apporter des éléments d'explication aux écarts constatés entre les bornes supérieures théoriques des pressions de soutènement critiques obtenues par DAVIS et al. (1980) et celles mesurées en centrifugeuse,

\section{BIBLIOGRAPHIE}

BROMS B.B., BENNERMARK H. (1967), Stability of clay at vertical openings. Journal of the Soil Mechanics and Foundations Division, ASCE, SM1 January 1967, pp. 71-94.

DAVIS E.H., GUNN M.J., MAIR R.J., SENEVIRATNE, H.N. (1980), The stability of shallow tunnels and underground openings in cohesive material. Géotechnique, 30, n 4 , pp. 397-416.

LECA E., PANET M. (1988), Application du calcul à la rupture à la stabilité du front de taille d'un tunnel. Revue Française de Géotechnique, $n^{\circ} 4$, pp. 5-19.

PECKER A., SALENÇON J. (1991), Seismic bearing capacity of shallow strip foundations on clay soils. CEE-Mexico International Workshop, 22-26/04/1991.

PECKER A., SALENÇON J. (1992), Capacité portante de fondations superficielles sous charges inclinées et excentrées. Journée "Problèmes scientifiques de l'ingénieur ». Palaiseau, 16 janvier 1992.

SALENÇON J. (1983), Calcul à la rupture et analyse limite. Presses de l'Ecole Nationale des Ponts et Chaussées, Paris, 366 pp.

SALENÇON J. (1990), An introduction to the yield design theory and its application to soil mechanics. European Journal of Mechanics, A/Solids 9. $n^{\circ}$ 5. pp. 277-288.

SCHOFIELD A.N. (1980), Cambridge geotechnical centrifuge operations. Géotechnique, $30, n^{\circ} 3$, 1980, pp. $227-268$.

\section{ANNEXE}

Soit $\underline{U}$ un champ de vitesses virtuel défini sur le système matériel $\Omega$. L'expression mathématique de la puissance résistante maximale est la suivante:

$\left.\mathrm{P}_{\max }^{\mathrm{res}}=\int_{\Omega} \pi(\underline{\underline{\mathrm{d}}}) \mathrm{d} \Omega+\int_{\Sigma} \pi(\underline{n}, \underline{U}]\right) \mathrm{d} \Sigma$ 
où $\Sigma$ désigne les surfaces de discontinuité éventuelles de $\underline{\mathrm{U}},[\underline{\mathrm{U}}], \underline{\mathrm{n}}$ et $\stackrel{\mathrm{d}}{\underline{\mathrm{d}}}$ désignent respectivement la discontinuité en question, la normale unitaire à $\Sigma$ et le taux de déformation dans le mécanisme envisagé. Les fonctions $\pi$ introduites dans (A1) sont définies de la manière suivante à partir du domaine $\mathrm{G}$ des contraintes admissibles :

$$
\begin{aligned}
& \pi(\underline{\underline{\mathrm{d}}})=\sup (\underline{\underline{\sigma}}: \underline{\underline{\mathrm{d}}}, \underline{\underline{\sigma}} \in \mathrm{G}) \\
& \pi(\underline{n},[\underline{U}])=\sup ([\underline{U}] \cdot \underline{\underline{\sigma}} \cdot \underline{\mathrm{n}}, \underline{\underline{\sigma}} \in \mathrm{G}) \text { (A2) }
\end{aligned}
$$

